\title{
Variação espacial da resposta do milho à adubação nitrogenada de cobertura em lavoura no cerrado
}

\author{
Sandro Manuel Carmelino Hurtado(1), Álvaro Vilela de Resende(2), Carlos Alberto Silva(1), \\ Edemar Joaquim Corazza ${ }^{(3)}$ e Luciano Shozo Shiratsuchi( ${ }^{(4)}$
}

\begin{abstract}
(1)Universidade Federal de Lavras, Caixa Postal 3037, CEP 37200-000 Lavras, MG. E-mail: sandroelbat@yahoo.com.br, csilva@ufla.br (2) Embrapa Milho e Sorgo, MG 424, Km 45, Caixa Postal 285, CEP 35701-970 Sete Lagoas, MG. E-mail: alvaro@cnpms.embrapa.br (3) Embrapa Informação Tecnológica, PqEB s/no, W3 (final), CEP 70770-901 Brasília, DF. E-mail: edemar@sct.embrapa.br ${ }^{(4)}$ Embrapa Cerrados, BR 020, Km 18, Caixa Postal 08223, CEP 73310-970 Planaltina, DF. E-mail: shozo@cpac.embrapa.br
\end{abstract}

Resumo - O objetivo deste trabalho foi avaliar, numa perspectiva espacial, a resposta do milho (Zea mays) à adubação de cobertura com nitrogênio $(\mathrm{N})$ e relacionar a produtividade de grãos com variáveis indicadoras do suprimento desse nutriente. Quatro doses de $\mathrm{N}$ foram testadas em 12 parcelas experimentais de 12,6x1.200 m. Em cada parcela foram georreferenciados 11 locais onde foram feitas as avaliações. Nesses locais, foi monitorado o estado nutricional do milho com o clorofilômetro e foram determinados os teores de $\mathrm{N}$ mineral do solo e os teores de $\mathrm{N}$ na folha e nos grãos. A produtividade de grãos foi mapeada com sensor de produtividade e "Global Positioning System" (GPS) acoplados à colhedora. Os dados foram analisados por estatística clássica e espacial. O cultivo sem aplicação de $\mathrm{N}$ em cobertura proporcionou, em média, $77 \%$ da máxima produtividade de milho $\left(9,21 \mathrm{Mg} \mathrm{ha}^{-1}\right)$ obtida com a adubação de cobertura. Altas correlações entre leitura do clorofilômetro, teor foliar de $\mathrm{N}$ e produtividade do milho, verificadas na análise de médias, não se confirmaram nos mapas que representam a variabilidade espacial dessas variáveis. A interpretação conjunta dos mapas de leitura do clorofilômetro e de produtividade do milho permitiu identificar áreas com diferentes capacidades de suprimento de $\mathrm{N}$ pelo solo e subsidiar a delimitação de zonas para o manejo diferenciado do nitrogênio.

Termos para indexação: Zea mays, agricultura de precisão, clorofilômetro, escala de campo, manejo sítio-específico, nitrogênio.

\section{Spatial variation of corn response to nitrogen topdressing in a Cerrado crop field}

\begin{abstract}
The objective of this work was to evaluate the spatial variation of corn response to nitrogen $(\mathrm{N})$ topdressing fertilization, associating the grain yield with indicative variables of the $\mathrm{N}$ nutritional status. Four $\mathrm{N}$ rates were tested in 12 experimental plots of $12.6 \times 1,200 \mathrm{~m}$. Along each plot, 11 georeferenced sites were located for punctual evaluations. In those sites, the corn nutritional status was monitored using a chlorophyll meter and samples were collected to determine soil mineral $\mathrm{N}$, and $\mathrm{N}$ concentration in leaves and grains. The grain yield was mapped using a yield sensor and a Global Positioning System (GPS) device coupled to the combine. The data obtained were analyzed using classical and spatial statistics. The corn with no $\mathrm{N}$ topdressing reached an average of $77 \%$ of the maximum grain yield $\left(9.21 \mathrm{Mg} \mathrm{ha}^{-1}\right)$ obtained with the usage of topdressing. The high correlation coefficients among the average data of chlorophyll meter readings, leaf $\mathrm{N}$ content, and grain yield were not confirmed when the spatial variability of these variables were considered. The map interpretation of the chlorophyll meter readings and corn yield allowed the identification of areas with different soil $\mathrm{N}$ supply capacity, indicating field zones for site-specific management.
\end{abstract}

Index terms: Zea mays, precision agriculture, chlorophyll meter, field-scale, site-specific management, nitrogen.

\section{Introdução}

$\mathrm{O}$ nitrogênio $(\mathrm{N})$ é um dos nutrientes mais limitantes para a obtenção de altas produtividades de milho, e as recomendações de adubação dependem de diversos fatores, como estande de plantas, extração/exportação do nutriente pela cultura, capacidade de suprimento de $\mathrm{N}$ pelo solo e índice de aproveitamento do fertilizante nitrogenado pelas plantas (Sousa \& Lobato, 2004).

Os critérios para definir a quantidade de $\mathrm{N}$ a ser fornecida costumam ser baseados na expectativa de rendimento, no histórico da área, no tipo de solo, no

Pesq. agropec. bras., Brasília, v.44, n.3, p.300-309, mar. 2009 
teor de matéria orgânica, nos teores de $\mathrm{N}$ mineral ou potencialmente mineralizável, na cultura antecedente e na utilização ou não de adubos verdes (Amado et al., 2002; Cantarella \& Duarte, 2004). O uso de irrigação (Souza et al., 2003), a densidade de plantas adotada (Amaral Filho et al., 2005) e a relação $\mathrm{C} / \mathrm{N}$ da cultura anterior (Vitti et al., 2003) também influenciam na determinação da dose ótima do nutriente.

$\mathrm{O}$ milho requer cerca de $20 \mathrm{~kg} \mathrm{ha}^{-1}$ de $\mathrm{N}$ para cada tonelada de grãos produzida (Fancelli, 2000; Sousa \& Lobato, 2004). Contudo, a máxima eficiência agronômica pode não coincidir com esse valor, como foi evidenciado por Silva et al. (2005). No Brasil, as recomendações regionais de $\mathrm{N}$ em cobertura apresentam variações, com indicação de doses que variam de 20 a $180 \mathrm{~kg} \mathrm{ha}^{-1}$ (Raij et al., 1996; Alves et al., 1999; Sousa \& Lobato, 2004). Essa variação reflete a diversidade de ambientes nos quais o milho é cultivado no país e a complexidade dos fatores que, em combinações variadas, influenciam a disponibilidade de $\mathrm{N}$ no solo e a eficiência das adubações.

A consideração das condições médias da lavoura para definição da adubação leva ao aparecimento de locais com sub ou superfertilização, o que pode resultar em variações de até $50 \%$ na produtividade de grãos dentro do talhão (Kitchen et al., 1995). Variação espacial pode ser observada em fatores relacionados ao suprimento de $\mathrm{N}$ pelo solo, como, por exemplo, nos teores de nitrato e de matéria orgânica (Ferguson et al., 2002; Machado et al., 2004) e de N potencialmente mineralizável (Mahmoudjafari et al.,1997), o que tem reflexo na quantidade de fertilizante nitrogenado a ser suprida às plantas. Aplicações de quantidades diferenciadas de fertilizantes nitrogenados, ponderadas pela variabilidade espacial do talhão, podem levar à maior eficiência de aproveitamento do $\mathrm{N}$ do solo $\mathrm{e}$ prevenir perdas de fertilizantes e riscos ao ambiente.

Dada a interferência de diversos fatores ligados ao clima, relevo, tipo de solo, manejo, genótipo e suas interações, não é simples predizer a quantidade de $\mathrm{N}$ requerida pelas plantas nem a resposta do milho à fertilização. Mesmo havendo variabilidade espacial da produtividade, existem entraves à aplicação do $\mathrm{N}$ em taxas variáveis (Welsh et al., 2003). Para isso torna-se necessário a obtenção de informações que possibilitem melhor reconhecimento dos principais fatores condicionantes da resposta à fertilização em âmbito local, ou seja, em cada talhão de cultivo.
Neste trabalho objetivou-se avaliar, numa perspectiva espacializada, a resposta do milho a doses de $\mathrm{N}$ em cobertura e relacionar a produtividade de grãos com variáveis indicadoras do suprimento de $\mathrm{N}$ em lavoura no Cerrado.

\section{Material e Métodos}

O estudo foi conduzido na safra 2006/2007 em lavoura de sequeiro na Fazenda Alto Alegre, Planaltina, GO. O experimento foi locado num talhão cultivado com sucessão soja-milho, sem preparo do solo há cinco anos, que apresentava ocorrência expressiva de Brachiaria spp. e outras gramíneas, as quais serviam ao pastejo bovino nos períodos de pousio, na estação seca. A produtividade média de grãos nas três safras anteriores foi de $3,1,8,8$ e $3,5 \mathrm{Mg} \mathrm{ha}^{-1}$, em cultivos de soja, milho e soja, respectivamente.

O solo, classificado como Latossolo VermelhoAmarelo, apresentou composição granulométrica média de $547 \mathrm{~g} \mathrm{~kg}^{-1}$ de argila, $149 \mathrm{~g} \mathrm{~kg}^{-1}$ de silte e $304 \mathrm{~g} \mathrm{~kg}^{-1}$ de areia, e os seguintes atributos químicos na camada de $0,0-0,2 \mathrm{~m}$, antes da semeadura do milho: matéria orgânica, $24 \mathrm{~g} \mathrm{~kg}^{-1} ; \mathrm{pH}_{\text {agua }} 5,7 ; \mathrm{P}_{\text {Mehlich }}, 6,1 \mathrm{mg} \mathrm{dm}{ }^{-3} ; \mathrm{K}$, $5 \mathrm{mmol}_{\mathrm{c}} \mathrm{dm}^{-3} ; \mathrm{Ca}, 21 \mathrm{mmol}_{\mathrm{c}} \mathrm{dm}^{-3} ; \mathrm{Mg}, 7 \mathrm{mmol}_{\mathrm{c}} \mathrm{dm}^{-3}$; CTC, $65 \mathrm{mmol}_{\mathrm{c}} \mathrm{dm}^{-3}$ e V, $50 \%$.

O híbrido simples de milho Pioneer 30F90 foi semeado em 16 de novembro de 2006, com aproximadamente 68.000 sementes por hectare. Na adubação realizada na semeadura foram aplicados $350 \mathrm{~kg} \mathrm{ha}^{-1}$ do formulado 6-23-18, que continha 2, 4, 0,13, 0,09, 0,13 e 0,4\% de Ca, S, B, Cu, Mn e Zn, respectivamente. Portanto, foram fornecidos $21,80 \mathrm{e}$ $63 \mathrm{~kg} \mathrm{ha}^{-1}$ de N, $\mathrm{P}_{2} \mathrm{O}_{5}$ e $\mathrm{K}_{2} \mathrm{O}$, respectivamente. O regime hídrico foi favorável durante o período experimental, com precipitações pluviais bem distribuídas.

Foram estudadas quatro doses de $\mathrm{N}$ em cobertura $\left(0,100,200\right.$ e $\left.300 \mathrm{~kg} \mathrm{ha}^{-1}\right)$, em delineamento de blocos ao acaso, com três repetições. Cada parcela compreendeu 18 fileiras espaçadas de $0,7 \mathrm{~m}$, com $1.200 \mathrm{~m}$ de comprimento, abrangendo $15.120 \mathrm{~m}^{2}$, numa área experimental total de 18,1 ha. $\mathrm{O}$ experimento foi conduzido com os procedimentos operacionais rotineiros da fazenda.

A adubação de cobertura iniciou-se quando as plantas apresentavam de três a quatro folhas desenvolvidas, no estádio fenológico $\mathrm{V}_{3}-\mathrm{V}_{4}$ (Ritchie et al., 2003), com uso de $100 \mathrm{~kg} \mathrm{ha}^{-1}$ de $\mathrm{N}$, correspondente ao manejo praticado na fazenda. Essa adubação foi 
complementada com a aplicação de $100 \mathrm{~kg} \mathrm{ha}^{-1}$ no estádio $V_{5}-V_{6}$ nos tratamentos com 200 e $300 \mathrm{~kg} \mathrm{ha}^{-1}$ e de $100 \mathrm{~kg} \mathrm{ha}^{-1}$ no estádio $\mathrm{V}_{6}-\mathrm{V}_{7}$ no tratamento com $300 \mathrm{~kg} \mathrm{ha}{ }^{-1}$. O fertilizante foi incorporado a $5 \mathrm{~cm}$ no solo e consistiu de uma mistura comercial (1:1) de grânulos de ureia e sulfato de amônio (33\% de N e $11 \%$ de S), sendo a ureia revestida com inibidor de urease $\mathrm{N}$-(n-butil) triamida tiofosfórica (NBPT).

$\mathrm{O}$ estado nutricional das plantas em relação ao $\mathrm{N}$ foi monitorado com o clorofilômetro Minolta SPAD 502 nos estádios fenológicos $\mathrm{V}_{8}$ e $\mathrm{R}_{1}$. Para cada estádio e parcela experimental, foram obtidas leituras SPAD nas seis linhas centrais em 11 locais de avaliação georreferenciados, distanciados $100 \mathrm{~m}$ entre si, ao longo do comprimento da parcela. Em cada local, dez leituras foram realizadas em cinco plantas escolhidas aleatoriamente em um raio de $2 \mathrm{~m}$, sendo uma leitura de cada lado do limbo foliar, no terço médio da folha, sem considerar a nervura central e as margens (Argenta et al., 2001). Avaliaram-se a primeira folha totalmente expandida a partir do ápice da planta, no estádio $\mathrm{V}_{8}$, e a folha abaixo e oposta à espiga, no estádio $R_{1}$.

No florescimento (estádio $\mathrm{R}_{1}$ ), em cada um dos 11 locais de cada parcela experimental foram coletadas cinco subamostras de solo na profundidade de 0,0-0,2 m, para constituir uma amostra composta, utilizada para determinação de amônio e nitrato (Tedesco et al., 1995). Nessa ocasião, coletaramse também as folhas utilizadas nas leituras SPAD, para análise do teor total de $\mathrm{N}$ segundo metodologia descrita em Malavolta et al. (1997). Com uso dessa metodologia, também se determinou o teor de $\mathrm{N}$ nos grãos, em amostras obtidas durante a colheita mecanizada das seis linhas centrais das parcelas experimentais, nos mesmos locais previamente georreferenciados.

Para obtenção da produtividade espacializada no talhão, utilizou-se uma colhedora com plataforma de seis linhas, equipada com o monitor PF Advantage com sensor de umidade e de fluxo de grãos e GPS (Teejet e-dif), com precisão de $2 \mathrm{~m}$ e correção de sinal por algoritmo interno. A umidade foi ajustada para $130 \mathrm{~g} \mathrm{~kg}^{-1}$. A produtividade final de grãos nas parcelas foi obtida mediante a remoção de dados dos pontos com erros de posicionamento, de dados com intervalos de distância entre pontos nulos, de dados de produtividade ou de umidade nulos e de dados discrepantes, conforme proposto por Menegatti \& Molin (2004).
As produtividades nos 11 locais de avaliação de cada parcela foram estimadas considerando-se a média dos dados da colhedora em um raio de $12 \mathrm{~m}$ do ponto georreferenciado.

Foram efetuadas análises estatísticas clássica e espacial.Utilizando-se conceitos da estatística clássica, os dados das variáveis estudadas foram submetidos às análises descritivas e de variância, por meio do uso do SISVAR (Ferreira, 2003), determinando-se modelos de regressão lineares e quadráticos para as variáveis de interesse. A estimativa da dose econômica de $\mathrm{N}$ foi feita com base nas cotações da saca de $60 \mathrm{~kg}$ de milho ( $\mathrm{R} \$ 24,00)$ e da tonelada do fertilizante nitrogenado (R\$ 933,00) em maio de 2007, não sendo considerados os custos relativos às operações de aplicação de $\mathrm{N}$ em cobertura. Coeficientes de correlação linear de Pearson foram obtidos com o SAS (SAS Institute, 2000).

$\mathrm{Na}$ análise espacial, para cada uma das quatro doses de $\mathrm{N}$ testadas foram processados os dados de leitura SPAD nos estádios $\mathrm{V}_{8}$ e $\mathrm{R}_{1}$ e os teores de $\mathrm{N}$ mineral no solo e $\mathrm{N}$ foliar obtidos nos locais de avaliação georreferenciados, com uso do programa geoestatístico VESPER (Minasny et al., 2002). A análise considerou a existência de isotropia. No ajuste dos modelos de semivariograma, foi considerada a menor soma de quadrados dos erros ou o menor critério de informação de Akaike (Webster \& McBratney, 1989), escolhendose, posteriormente, aquele com média e variância do erro mais próxima de zero e um, respectivamente, por meio da autovalidação (Vieira, 2000). Na sequência, foi realizada a interpolação por krigagem. O grau de dependência espacial foi obtido segundo Cambardella et al. (1994). Mapas foram confeccionados, após interpolação, com uso do programa ArcView 3.2, considerando os dados da krigagem para leitura SPAD no estádio $\mathrm{V}_{8}$ e teor de $\mathrm{N}$ foliar. $\mathrm{O}$ mapa de produtividade de grãos foi confeccionado com os valores reais.

\section{Resultados e Discussão}

As doses de $\mathrm{N}$ influenciaram significativamente as variáveis em estudo e os dados, na maioria dos casos, ajustaram-se a modelos quadráticos (Figura 1). A estimativa da produtividade máxima de grãos $\left(9,2 \mathrm{Mg} \mathrm{ha}^{-1}\right)$ correspondeu à aplicação da dose de $242 \mathrm{~kg} \mathrm{ha}^{-1}$ de N. Já a dose econômica, de acordo com a relação de preços do fertilizante e do produto colhido, foi de $143 \mathrm{~kg} \mathrm{ha}^{-1}$ (Figura $1 \mathrm{~A}$ ). A diferença 
nas médias de produtividade obtidas com ou sem a adubação de cobertura foi relativamente baixa. A mesma tendência foi verificada para o acúmulo de $\mathrm{N}$ nos grãos (Figura $1 \mathrm{~A}$ ).

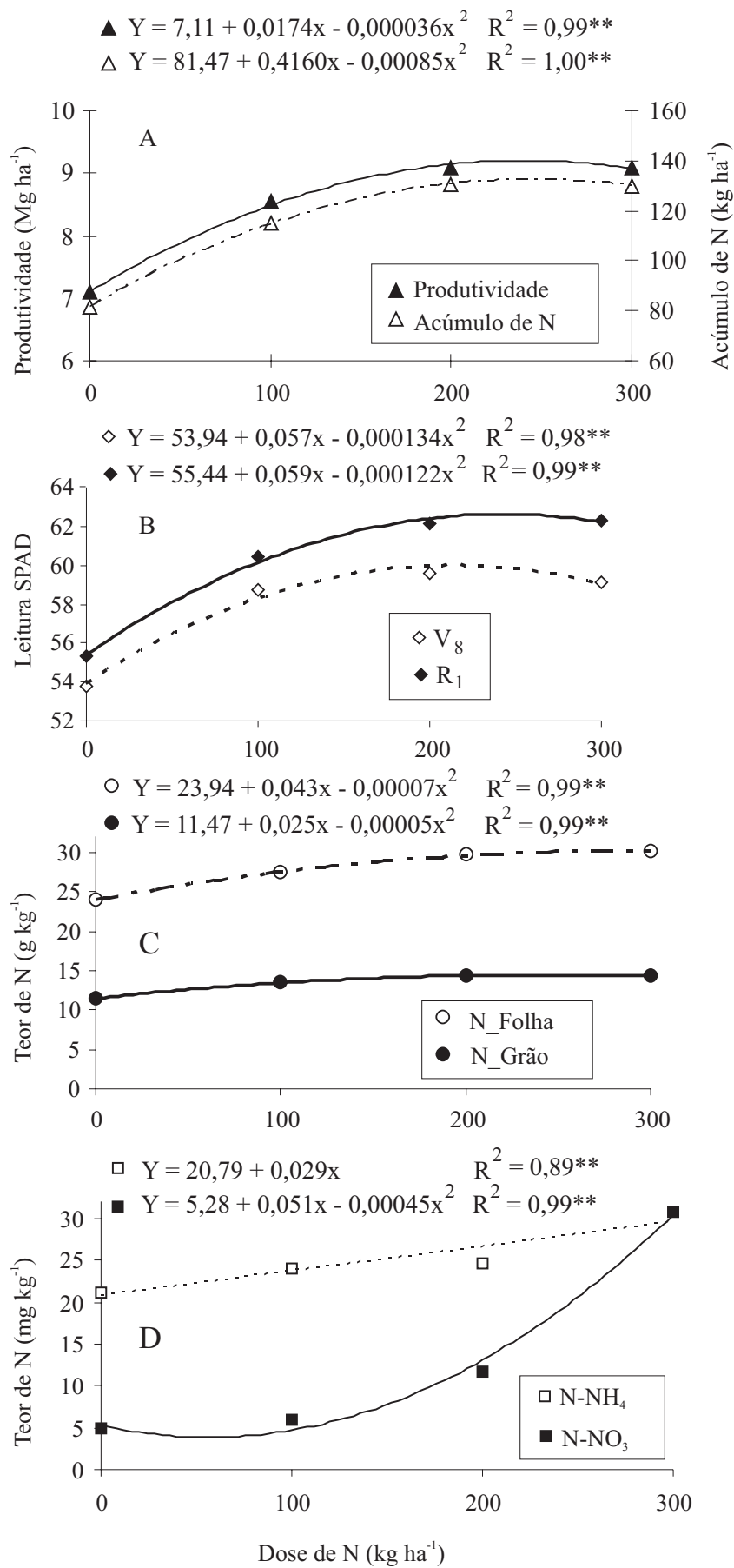

Figura 1. Dados médios de produtividade e acúmulo de $\mathrm{N}$ nos grãos (A), leituras SPAD nos estádios $\mathrm{V}_{8}$ e $\mathrm{R}_{1}(\mathrm{~B})$, teores de $\mathrm{N}$ foliar e nos grãos (C) e teores de amônio e nitrato no solo (D), em função de doses de $\mathrm{N}$ na adubação de cobertura do milho.
A resposta pouco expressiva do milho à adubação nitrogenada de cobertura está de acordo com a produtividade elevada observada no tratamento sem $\mathrm{N}$ em cobertura $\left(7,11 \mathrm{Mg} \mathrm{ha}^{-1}\right)$ e indica que o solo possuía um bom estoque natural de $\mathrm{N}$ e que as condições edafoclimáticas foram propícias à mineralização. $\mathrm{O}$ tratamento sem cobertura recebeu $21 \mathrm{~kg} \mathrm{ha}^{-1}$ de $\mathrm{N}$ na semeadura e o sistema de sucessão com soja favorece um bom suprimento desse nutriente, comparativamente ao monocultivo de milho (Sousa \& Lobato, 2004), o que resulta em um menor potencial de resposta à adubação nitrogenada. Below (2002) relatou a contribuição da cultura da soja na redução da dose de $\mathrm{N}$ necessária à otimização da produtividade do milho em um solo com alto potencial produtivo no estado de Illinois, EUA. Sob cultivo contínuo do milho, esse autor constatou que houve resposta a até $235 \mathrm{~kg} \mathrm{ha}^{-1}$ de $\mathrm{N}$, ao passo que, na sucessão soja/milho, o potencial de rendimento de grãos de milho foi atingido com cerca de $135 \mathrm{~kg} \mathrm{ha}^{-1}$ de N. Além disso, há maior eficiência na utilização do $\mathrm{N}$ pelo milho em condições de baixo suprimento do nutriente (Souza et al., 2008), o que ajuda a justificar a elevada produtividade observada no tratamento sem adubação de cobertura.

De acordo com os modelos ajustados (Figura $1 \mathrm{~A}$ ), a remoção de $\mathrm{N}$ com a colheita foi de $81,5 \mathrm{~kg} \mathrm{ha}^{-1}$ no tratamento sem cobertura nitrogenada. Considerandose que o $\mathrm{N}$ exportado nos grãos corresponde a $75 \%$ do que é extraído pela cultura (Vitti et al., 2003) e descontados os $21 \mathrm{~kg}$ fornecidos na adubação de semeadura, pode-se estimar que o solo supriu cerca de $87,6 \mathrm{~kg} \mathrm{ha}^{-1}$ de $\mathrm{N}$ durante o ciclo do milho.

Os teores relativos de clorofila (leituras SPAD) mais elevados foram detectados no estádio $\mathrm{R}_{1}$ (Figura $1 \mathrm{~B}$ ), o que corrobora os resultados observados por Argenta et al. (2004). De acordo com os modelos, o potencial metabólico de produção de clorofila pelo híbrido de milho utilizado (ponto de máxima resposta) em relação ao fornecimento de $\mathrm{N}$ foi alcançado com as doses de 213 e $242 \mathrm{~kg} \mathrm{ha}^{-1}$ de $\mathrm{N}$ nos estádios $\mathrm{V}_{8}$ e $\mathrm{R}_{1}$, respectivamente. Portanto, as doses de $\mathrm{N}$ para máxima síntese de clorofila no florescimento e para máxima produção de grãos $\left(242 \mathrm{~kg} \mathrm{ha}^{-1}\right)$ foram coincidentes. As maiores leituras do clorofilômetro foram de 60,0 e 62,6 unidades SPAD em $V_{8}$ e $R_{1}$, respectivamente, enquanto os valores relacionados à produtividade econômica foram de 59,4 e 61,4 nos referidos estádios fenológicos. 
A partir da dose de $100 \mathrm{~kg} \mathrm{ha}^{-1}$ de $\mathrm{N}$, os teores foliares de N (Figura $1 \mathrm{C}$ ) enquadraram-se na faixa de 28-35 $\mathrm{g} \mathrm{kg}^{-1}$, considerada adequada para a cultura (Oliveira, 2004). Os teores associados às produtividades máxima e econômica foram de 30 e $29 \mathrm{~g} \mathrm{~kg}^{-1}$, respectivamente. Já o teor de $\mathrm{N}$ nos grãos não apresentou incremento expressivo com a adubação de cobertura, com valor máximo de $14 \mathrm{~g} \mathrm{~kg}^{-1}$, o qual coincide com o valor médio relatado por Fancelli (2000).

Em relação ao $\mathrm{N}$ mineral no solo no estádio de florescimento (Figura 1 D), verificou-se aumento dos teores, principalmente dos de nitrato, a partir da dose de $100 \mathrm{~kg} \mathrm{ha}^{-1}$ de $\mathrm{N}$. O acúmulo de $\mathrm{N}$ no solo, associado às respostas quadráticas do teor foliar e acúmulo do $\mathrm{N}$ nos grãos, sugere que não deve ter havido consumo de luxo. Ao contrário, a partir da dose de $200 \mathrm{~kg} \mathrm{ha}^{-1}$ de $\mathrm{N}$ parece ter-se atingido o máximo da capacidade fisiológica de absorção do nutriente, que é uma característica que apresenta controle genético e varia de acordo com a cultivar utilizada (Souza et al., 2008). Resultados similares foram obtidos por Fernandes et al. (1999) que, ao avaliar doses de 0 a $240 \mathrm{~kg} \mathrm{ha}^{-1}$ de $\mathrm{N}$ em cobertura, para milho cultivado em um Latossolo Vermelho distrófico de Cerrado, não observaram grande aumento da absorção do nutriente nas maiores doses.

Os modelos da Figura 1 A e B mostram forte semelhança nas tendências das leituras SPAD, produtividade e acúmulo de $\mathrm{N}$ nos grãos por efeito das doses crescentes de $\mathrm{N}$ em cobertura, como se comprova pela alta correlação positiva entre médias dessas variáveis (Tabela 1). A existência de correlação significativa da produtividade com as leituras do clorofilômetro, a partir dos estádios $\mathrm{V}_{8}$ e $\mathrm{V}_{10}$, foi relatada por Varvel et al. (1997) e Argenta et al. (2004), respectivamente. Argenta et al. (2002) destacaram a possibilidade de se inferir sobre o estado nutricional de $\mathrm{N}$ em milho a partir de leituras de clorofilômetro. Contudo, a viabilidade de uso desse equipamento para diagnosticar deficiência nutricional e necessidade de adubação nitrogenada parece ser tanto maior quanto mais contrastantes forem as condições de suprimento de $\mathrm{N}$ em diferentes locais da lavoura.

Não se obteve correlação significativa do teor de $\mathrm{N}$ mineral (amônio mais nitrato) no solo, no estádio de florescimento, com as demais variáveis apresentadas na Tabela 1. Ao estudar os teores de $\mathrm{N}$ mineral no solo como indicadores da disponibilidade do nutriente para o milho, Rambo et al. (2007) encontraram boa correlação entre produtividade e os teores de amônio e nitrato no solo, determinados no estádio $\mathrm{V}_{6}$, o que não se verificou para as determinações realizadas em $\mathrm{V}_{10} \mathrm{e}$ no espigamento.

Ao se estabelecer a relação entre as leituras SPAD e a produtividade, a partir dos dados amostrais dos 11 locais, foram observados distintos padrões de resposta, conforme a dose de N (Figura 2). Houve maior dispersão de pontos na ausência da adubação de cobertura, o que indica a ocorrência de variabilidade espacial nos atributos do solo relacionados à disponibilidade de $\mathrm{N}$ às plantas. Nessa condição, as leituras do clorofilômetro podem auxiliar na tomada de decisão sobre a necessidade, o local e o momento de realizar a adubação de cobertura. Todavia, com o fornecimento do nutriente (doses 100 a $300 \mathrm{~kg} \mathrm{ha}^{-1}$ ), a tendência de aglutinação dos pontos na Figura 2 indica situação em que fica mascarada a influência da capacidade original de suprimento de $\mathrm{N}$ pelo solo e a sua variabilidade espacial.

A ocorrência de variabilidade espacial pode ser inferida, também, a partir de correlações entre os dados amostrais dos 11 locais, distribuídos ao

Tabela 1. Coeficientes de correlação (r) entre leitura SPAD nos estádios $\mathrm{V}_{8}$ e $\mathrm{R}_{1}, \mathrm{~N}$ mineral no solo, teor de $\mathrm{N}$ foliar, teor de $\mathrm{N}$ nos grãos, produtividade, acúmulo de $\mathrm{N}$ nos grãos e doses de $\mathrm{N}$ na cultura do milho. Cálculo a partir das médias das variáveis nas doses de $\mathrm{N}$ testadas $(\mathrm{n}=4)$.

\begin{tabular}{|c|c|c|c|c|c|c|c|}
\hline Variável & $\begin{array}{c}\text { SPAD } \\
\mathrm{R}_{1}\end{array}$ & $\begin{array}{l}\text { Nitrogênio } \\
\text { mineral }\end{array}$ & $\begin{array}{l}\text { Teor de } \\
\mathrm{N} \text { foliar }\end{array}$ & $\begin{array}{l}\text { Teor de } \mathrm{N} \\
\text { nos grãos }\end{array}$ & $\begin{array}{c}\text { Produtividade } \\
\text { de grãos }\end{array}$ & $\begin{array}{l}\text { Acúmulo de } \\
\mathrm{N} \text { nos grãos }\end{array}$ & $\begin{array}{l}\text { Dose } \\
\text { de N }\end{array}$ \\
\hline $\mathrm{SPAD} \mathrm{V}_{8}{ }^{(1)}$ & $0,98^{*}$ & $0,54^{\text {ns }}$ & $0,94 * *$ & $0,98^{*}$ & $0,98 *$ & $0,98^{*}$ & $0,81^{\text {ns }}$ \\
\hline SPAD R ${ }^{(2)}$ & & $0,67^{\mathrm{ns}}$ & $0,98 * *$ & $0,99 * *$ & $0,99 * *$ & $0,99 * *$ & $0,90^{\text {ns }}$ \\
\hline $\mathrm{N}$ mineral ${ }^{(3)}$ & & & $0,75^{\mathrm{ns}}$ & $0,66^{\mathrm{ns}}$ & $0,66^{\mathrm{ns}}$ & $0,66^{\mathrm{ns}}$ & $0,91^{\mathrm{ns}}$ \\
\hline Teor de $\mathrm{N}$ foliar & & & & $0,99 * *$ & $0,99 *$ & $0,99 * *$ & $0,95 *$ \\
\hline Teor de N nos grãos & & & & & $1,00 * *$ & $1,00 * *$ & 0,90 \\
\hline Produtividade & & & & & & $1,00 * *$ & $0,90^{\mathrm{ns}}$ \\
\hline Acúmulo de $\mathrm{N}$ nos grãos & & & & & & & $0,90^{\mathrm{ns}}$ \\
\hline
\end{tabular}

Pesq. agropec. bras., Brasília, v.44, n.3, p.300-309, mar. 2009 
longo de cada parcela (Tabela 2). Observa-se que os coeficientes de correlação são mais baixos que os apresentados na Tabela 1, o que indica inconstância no padrão de resposta da cultura ao fornecimento de $\mathrm{N}$, enquanto o que se esperava era um comportamento relativamente uniforme. Quando se trabalha com os valores médios das respostas às doses de $\mathrm{N}$ (Tabela 1) ignora-se a possibilidade de existência de variabilidade espacial dentro da parcela experimental. No entanto, há variação, em curtas distâncias, nos fatores que influenciam a disponibilidade de $\mathrm{N}$ no solo e de outros fatores ligados ao desenvolvimento e à produtividade do milho (Mahmoudjafari et al., 1997; Ferguson et al., 2002; Machado et al., 2004).

Os coeficientes de variação $(\mathrm{CV})$ da análise descritiva das leituras de SPAD nos estádios $\mathrm{V}_{8}$ e $\mathrm{R}_{1}$ e $\mathrm{N}$ foliar para cada dose de $\mathrm{N}$ foram baixos N. Para mineral no solo, os valores de $\mathrm{CV}$ podem ser considerados médios: maiores que $12 \%$ (Tabela 3 ). A distribuição dos dados foi simétrica para a maioria dos casos, evidenciada nos coeficientes de assimetria e curtose próximos de zero e três, respectivamente, assim como nos valores similares de média e mediana. Tal comportamento confirma a presença de normalidade (Carvalho et al., 2002).

$\mathrm{Na}$ análise geoestatística, a presença de estrutura espacial - ou da existência de aparente continuidade entre os locais - foi detectada para todas as variáveis, à exceção do $\mathrm{N}$ foliar na dose de $100 \mathrm{~kg} \mathrm{ha}^{-1}$ (Tabela 3). Foram determinados alcances efetivos que variaram de 116 a $755 \mathrm{~m}$. O grau de dependência espacial, estratificado em classes segundo a razão entre a variância não estruturada e a total (Cambardella et al., 1994), variou de fraco a moderado para as leituras SPAD, de fraco a forte para o $\mathrm{N}$ foliar, e de moderado a forte para o $\mathrm{N}$ mineral no solo. Graus
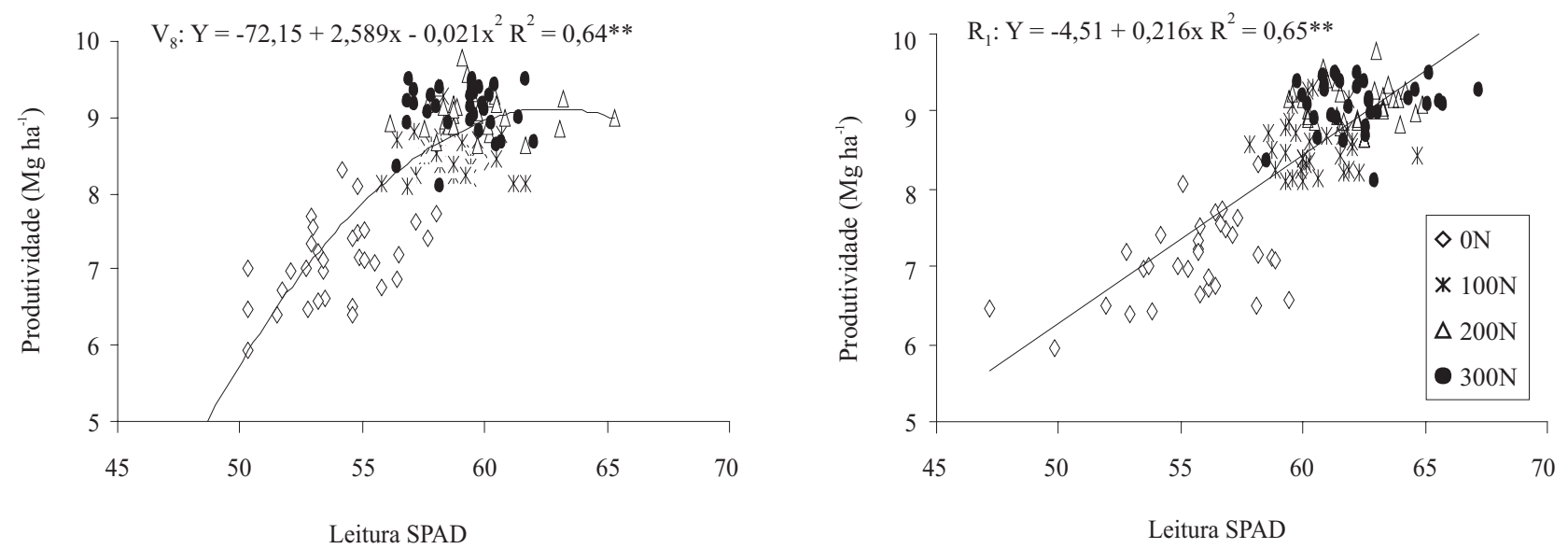

Figura 2. Relação entre leitura SPAD e produtividade de grãos de milho nos estádios $\mathrm{V}_{8}$ e $\mathrm{R}_{1}$ em resposta a doses de $\mathrm{N}$ $\left(\mathrm{kg} \mathrm{ha}^{-1}\right)$ em cobertura.

Tabela 2. Coeficientes de correlação (r) entre leitura SPAD nos estádios $\mathrm{V}_{8}$ e $\mathrm{R}_{1}$, $\mathrm{N}$ mineral no solo, teor de $\mathrm{N}$ foliar, teor de $\mathrm{N}$ nos grãos, produtividade, acúmulo de $\mathrm{N}$ nos grãos e doses de $\mathrm{N}$ aplicadas na cultura do milho. Cálculo a partir dos dados amostrais de 11 locais de avaliação em cada parcela experimental $(\mathrm{n}=132)$.

\begin{tabular}{|c|c|c|c|c|c|c|c|}
\hline Variável & $\begin{array}{c}\text { SPAD } \\
\mathrm{R}_{1}\end{array}$ & $\begin{array}{c}\text { Nitrogênio } \\
\text { mineral }\end{array}$ & $\begin{array}{l}\text { Teor de N } \\
\text { foliar }\end{array}$ & $\begin{array}{l}\text { Teor de N } \\
\text { nos grãos }\end{array}$ & $\begin{array}{c}\text { Produtividade } \\
\text { de grãos }\end{array}$ & $\begin{array}{l}\text { Acúmulo de } \\
\text { N nos grãos }\end{array}$ & $\begin{array}{l}\text { Dose } \\
\text { de N }\end{array}$ \\
\hline SPAD V $_{8}^{(1)}$ & $0,79 * *$ & $0,31 * *$ & $0,67 * *$ & $0,62 * *$ & $0,76 * *$ & $0,76 * *$ & $0,65 * *$ \\
\hline SPAD $\mathrm{R}_{1}^{(2)}$ & & $0,45 * *$ & $0,76^{* *}$ & $0,66^{* *}$ & $0,81 * *$ & $0,79 * *$ & $0,74 * *$ \\
\hline $\mathrm{N}$ mineral ${ }^{(3)}$ & & & $0,46 * *$ & $0,35 * *$ & $0,49 * *$ & $0,43 * *$ & $0,71 * *$ \\
\hline Teor de $\mathrm{N}$ foliar & & & & $0,61 * *$ & $0,74 * *$ & $0,72 * *$ & $0,77 * *$ \\
\hline Teor de $\mathrm{N}$ nos grãos & & & & & $0,68 * *$ & $0,89 * *$ & $0,81 * *$ \\
\hline Produtividade & & & & & & $0,90 * *$ & $0,81 * *$ \\
\hline Acúmulo de $\mathrm{N}$ nos grãos & & & & & & & $0,78 * *$ \\
\hline
\end{tabular}

(1)Leitura do clorofilômetro no estádio fenológico $\mathrm{V}_{8}$ do milho. ${ }^{(2)}$ Leitura do clorofilômetro no estádio fenológico $\mathrm{R}_{1}$ do milho. ${ }^{(3)}$ Amônio mais nitrato no solo. ** Significativo a $1 \%$ de probabilidade. 
de dependência espacial de moderado a forte foram relatados para o nitrato em solos cultivados com milho (Ferguson et al., 2002).

De maneira geral, os valores de alcance efetivo determinados não foram similares entre as doses de $\mathrm{N}$ (Tabela 3), não sendo possível assumir um padrão único de dependência espacial para uma dada variável. Isso dificulta a indicação de critérios baseados no alcance efetivo, para estratégias de mapeamento futuro, em grades regulares, na área em questão. Também não se encontrou indicativo consistente que pudesse servir de critério isolado para o estabelecimento de zonas de manejo de $\mathrm{N}$, conforme se pode visualizar nos mapas interpolados por krigagem a partir dos semivariogramas (Figura 3).

Observaram-se algumas variações de produtividade dentro de cada parcela experimental, porém sem um padrão de continuidade entre faixas (Figura 3). A pequena amplitude dos dados de produtividade, com baixa resposta às doses mais elevadas de $\mathrm{N}$, contribuiu para minimizar os contrastes.

Semelhança no comportamento espacial pode ser notada entre a resposta de produtividade do milho e as leituras $\mathrm{SPAD}$ em $\mathrm{V}_{8}$ (Figura 3), o que também ocorreu para as leituras em $\mathrm{R}_{1}$. Embora sensíveis à cobertura nitrogenada, as variações do teor de $\mathrm{N}$ foliar foram menos sincronizadas com a produtividade. Análises de correlação a partir de valores extraídos dos mapas interpolados, referentes aos 11 locais amostrais nas parcelas e feitas para cada dose de $\mathrm{N}$ separadamente (n = 33), mostraram alguma correspondência apenas entre a leitura SPAD nos estádios $V_{8}$ e $R_{1}$ e a produtividade de grãos na ausência de cobertura nitrogenada $\left(\mathrm{r}=0,36^{* *}\right.$ e $\mathrm{r}=0,40^{* *}$, respectivamente). As correlações que envolveram $\mathrm{N}$ foliar e $\mathrm{N}$ mineral no solo não foram significativas em nenhuma das doses estudadas.

Os elevados coeficientes obtidos na análise de correlação de médias (Tabela 1), indicativos de dependência da produtividade em relação à disponibilidade de $\mathrm{N}$ no sistema solo/planta, foram reduzidos quando se trabalhou com a abordagem espacializada. Portanto, em sistemas abertos e de larga escala (condições de lavoura) são menores as chances de sucesso das tentativas de integrar a variação espacial de indicadores da disponibilidade de $\mathrm{N}$ e a resposta produtiva do milho. Assim, deve ser considerada a possibilidade de validação, no Brasil, de outras técnicas que envolvam sensoriamento remoto (Povh et al., 2008), para detectar diferenças no estado nutricional das plantas, com vistas a aplicações de $\mathrm{N}$ em taxas variáveis.

As medidas do clorofilômetro foram o indicador que melhor refletiu o potencial de resposta do milho à adubação nitrogenada em cobertura, e permitiram identificar áreas com menor capacidade de suprimento de $\mathrm{N}$ pelo solo (Figura 3) e, consequentemente, com maior probabilidade de resposta à adubação de

Tabela 3. Estatística descritiva, parâmetros de ajuste dos semivariogramas e grau de dependência espacial (GDE) para as leituras SPAD nos estádios $\mathrm{V}_{8}$ e $\mathrm{R}_{1}$ da cultura do milho, nitrogênio mineral no solo e nitrogênio foliar.

\begin{tabular}{|c|c|c|c|c|c|c|c|c|c|c|c|c|c|}
\hline \multirow[t]{2}{*}{ Variável } & \multirow[t]{2}{*}{ Mínimo } & \multirow[t]{2}{*}{ Máximo } & \multirow[t]{2}{*}{ Mediana } & \multirow[t]{2}{*}{ Média } & \multirow{2}{*}{$\begin{array}{l}\mathrm{CV} \\
(\%)\end{array}$} & \multicolumn{2}{|c|}{ Coeficiente } & \multirow[t]{2}{*}{ Modelo } & \multirow{2}{*}{$\begin{array}{c}\text { Efeito pepita } \\
\text { (Co) }\end{array}$} & \multirow{2}{*}{$\begin{array}{c}\text { Contribuição } \\
\left(\mathrm{C}_{1}\right)\end{array}$} & \multirow{2}{*}{$\begin{array}{l}\text { Alcance } \\
\text { efetivo }\end{array}$} & \multirow[t]{2}{*}{$\mathrm{RMSE}^{(1)}$} & \multirow[t]{2}{*}{$\mathrm{GDE}^{(2)}$} \\
\hline & & & & & & Assimetria & Curtose & & & & & & \\
\hline & \multicolumn{13}{|c|}{$0 \mathrm{~N}$} \\
\hline $\mathrm{SPAD} \mathrm{V}_{8}$ & 48,1 & 58,0 & 53,5 & 53,8 & 4,2 & $-0,30$ & 3,00 & Gaussiano & 2,03 & 3,62 & 537,9 & 2,57 & Moderado \\
\hline SPAD R 1 & 47,2 & 59,4 & 55,8 & 55,3 & 4,8 & $-1,03$ & 4,18 & Gaussiano & 4,02 & 5,45 & 755,1 & $1,58 \quad$ & Moderado \\
\hline $\mathrm{N}$ mineral & 13,3 & 47,3 & 26,6 & 26,0 & 25,8 & 0,34 & 4,92 & Esférico & 7,95 & 42,15 & 364,3 & 1,32 & Forte \\
\hline \multirow[t]{2}{*}{$\underline{\mathrm{N} \text { foliar }}$} & 19,4 & 30,0 & 23,8 & 23,9 & 9,2 & 0,69 & 4,14 & Esférico & 0,44 & 4,39 & 174,1 & 1,36 & Forte \\
\hline & \multicolumn{13}{|c|}{$100 \mathrm{~N}$} \\
\hline $\mathrm{SPAD} \mathrm{V}_{8}$ & 55,8 & 61,7 & 58,6 & 58,7 & 2,3 & 0,06 & 2,75 & Esférico & 1,28 & 0,43 & 116,7 & 0,63 & Fraco \\
\hline SPAD R $_{1}$ & 57,8 & 64,7 & 60,2 & 60,5 & 2,2 & 0,75 & 4,03 & Exponencial & 0,54 & 1,39 & 175,1 & 0,67 & Moderado \\
\hline $\mathrm{N}$ mineral & 14,7 & 36,6 & 30,6 & 30,1 & 12,5 & $-2,01$ & 9,99 & Esférico & 8,10 & 10,80 & 275,9 & 6,46 & Moderado \\
\hline \multirow[t]{2}{*}{$\underline{\mathrm{N} \text { foliar }}$} & 24,7 & 30,4 & 27,2 & 27,4 & 4,9 & 0,31 & 2,50 & $\mathrm{EPP}^{(3)}$ & - & - & - & - & - \\
\hline & \multicolumn{13}{|c|}{$200 \mathrm{~N}$} \\
\hline $\mathrm{SPAD} \mathrm{V}_{8}$ & 56,1 & 65,3 & 59,3 & 59,6 & 3,0 & 1,20 & 5,04 & Exponencial & 2,79 & 0,48 & 205,9 & 0,88 & Fraco \\
\hline SPAD R 1 & 59,4 & 64,9 & 62,2 & 62,1 & 2,5 & $-0,04$ & 1,95 & Esférico & 1,28 & 1,11 & 234,2 & $0,48 \quad 1$ & Moderado \\
\hline $\mathrm{N}$ mineral & 23,3 & 52,6 & 36,0 & 36,4 & 17,5 & 0,41 & 3,22 & Esférico & 17,34 & 22,12 & 195,8 & 6,391 & Moderado \\
\hline \multirow[t]{2}{*}{$\underline{\mathrm{N} \text { foliar }}$} & 25,7 & 33,8 & 29,9 & 29,7 & 6,7 & $-0,13$ & 2,75 & Esférico & 1,37 & 2,03 & 257,5 & 1,93 & Moderado \\
\hline & \multicolumn{13}{|c|}{$300 \mathrm{~N}$} \\
\hline $\mathrm{SPAD} \mathrm{V}_{8}$ & 56,4 & 62,0 & 59,5 & 59,2 & 2,5 & $-0,19$ & 2,22 & Gaussiano & 1,49 & 0,91 & 317,1 & 0,60 & Moderado \\
\hline SPAD R 1 & 58,5 & 67,2 & 62,2 & 62,3 & 3,1 & 0,59 & 2,96 & Esférico & 2,84 & 0,74 & 230,0 & 1,75 & Fraco \\
\hline $\mathrm{N}$ mineral & 34,6 & 123,2 & 57,3 & 61,5 & 32,7 & 1,10 & 4,15 & Exponencial & 290,30 & 131,10 & 255,6 & $2,02 \quad$ & Moderado \\
\hline$\underline{\mathrm{N} \text { foliar }}$ & 26,9 & 32,8 & 30,1 & 30,1 & 4,8 & $-0,09$ & 2,39 & Esférico & 1,72 & 0,48 & 291,8 & 0,88 & Fraco \\
\hline
\end{tabular}

${ }^{(1)}$ Soma de quadrados dos erros. ${ }^{(2)}$ Grau de dependência espacial (Cambardella et al., 1994). ${ }^{(3)}$ Efeito pepita puro. 


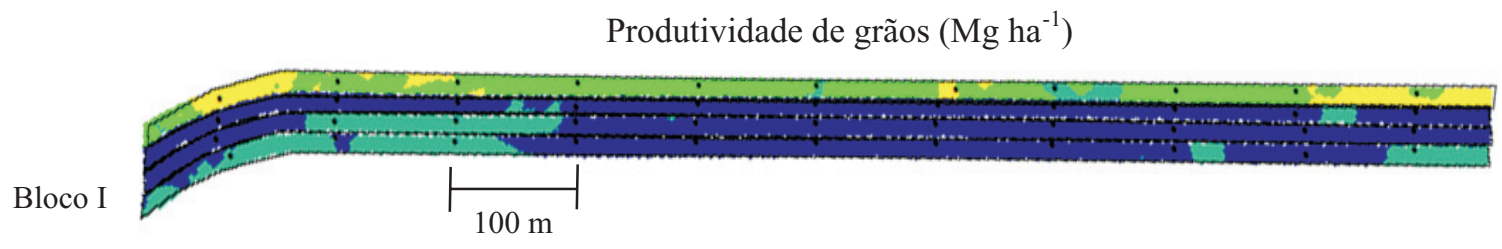

$00 \mathrm{~N}$
$200 \mathrm{~N}$
$300 \mathrm{~N}$

Bloco I

$100 \mathrm{~m}$
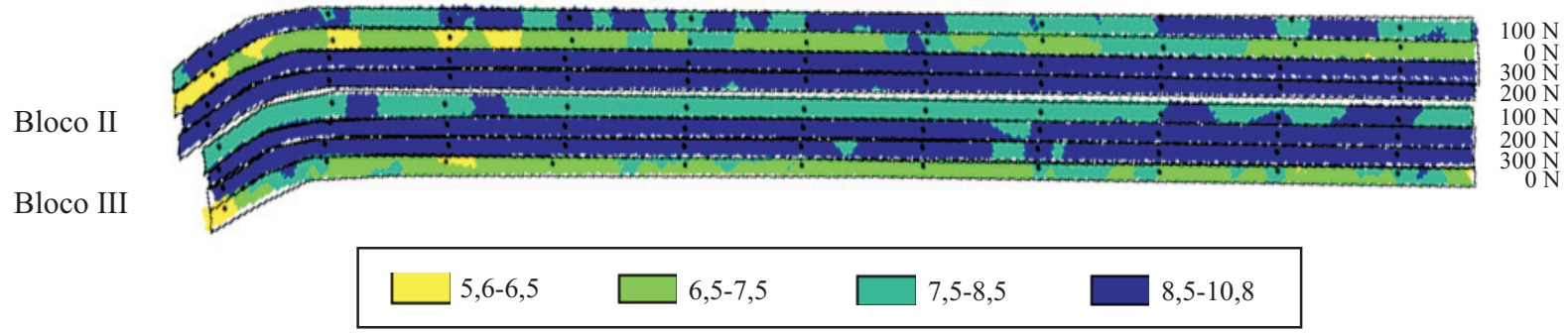

Leitura SPAD

Bloco I

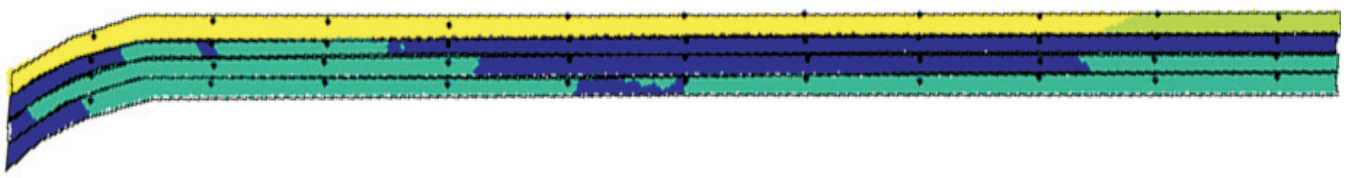

$200 \mathrm{~N}$

$300 \mathrm{~N}$

$100 \mathrm{~N}$

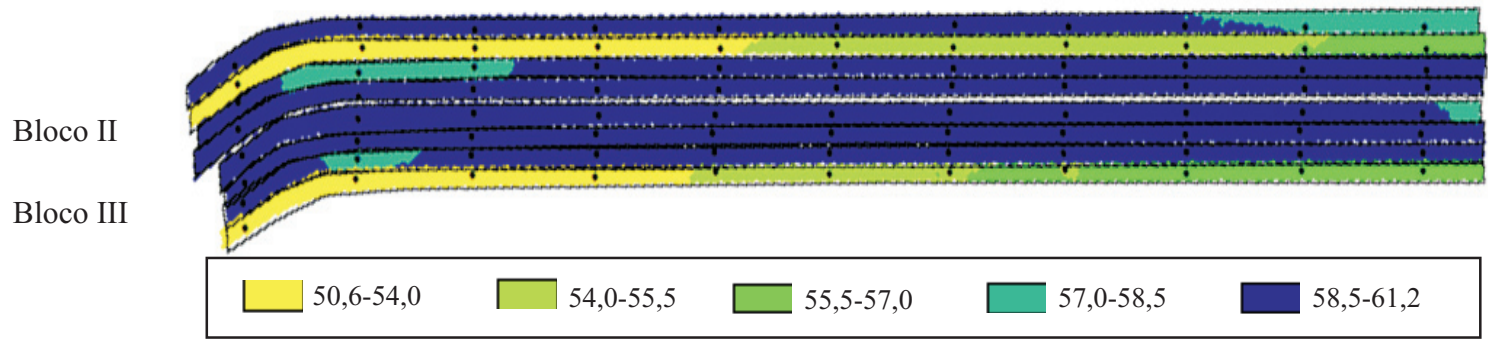

$100 \mathrm{~N}$

$0 \mathrm{~N}$
$300 \mathrm{~N}$
$200 \mathrm{~N}$

$200 \mathrm{~N}$

$200 \mathrm{~N}$

$300 \mathrm{~N}$
$0 \mathrm{~N}$

Bloco III

foliar $\left(\mathrm{g} \mathrm{kg}^{-1}\right)$

Bloco I

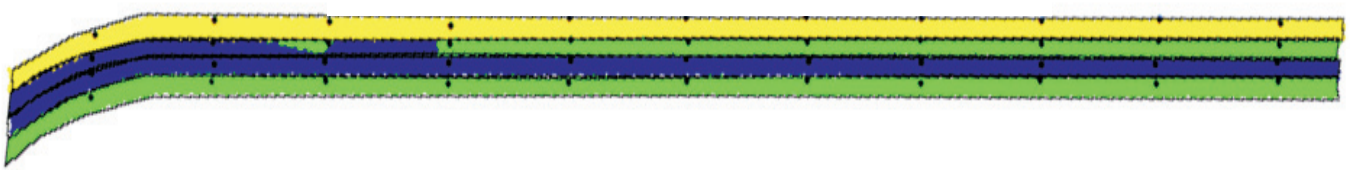

Bloco II

Bloco III

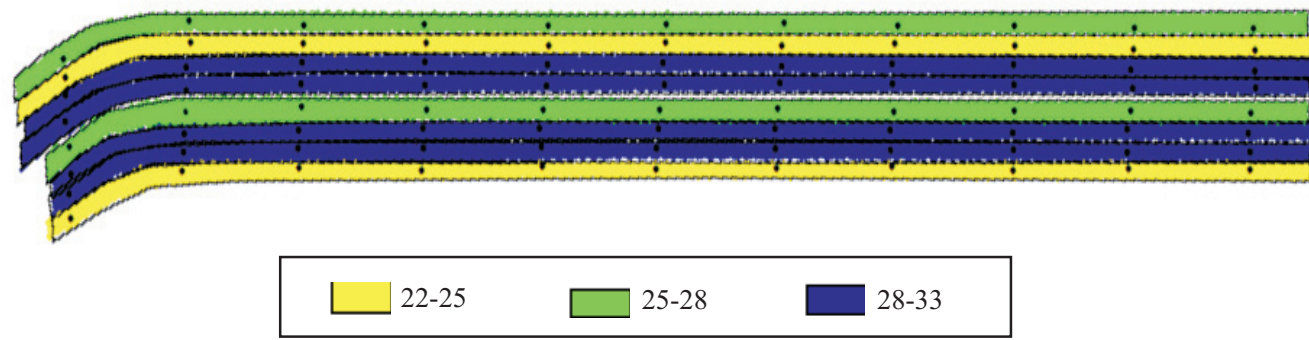

$100 \mathrm{~N}$
$0 \mathrm{~N}$
$300 \mathrm{~N}$
$200 \mathrm{~N}$
$100 \mathrm{~N}$
$200 \mathrm{~N}$
$300 \mathrm{~N}$
$0 \mathrm{~N}$

Figura 3. Mapas de produtividade de grãos de milho, de leitura SPAD no estádio $\mathrm{V}_{8}$ e de teor de $\mathrm{N}$ foliar, em resposta às doses de $0,100,200$ e $300 \mathrm{~kg} \mathrm{ha}^{-1}$ de $\mathrm{N}$ em cobertura. 
cobertura. Assim, a interpretação conjunta dos mapas de leitura SPAD e de produtividade (Figura 3) pode subsidiar a delimitação de zonas de manejo diferenciado da adubação nitrogenada do milho.

\section{Conclusões}

1. Altas correlações entre leitura do clorofilômetro, teor foliar de nitrogênio e produtividade do milho, verificadas na análise de médias, não se confirmam nos mapas que representam a variabilidade espacial dessas variáveis.

2. A interpretação conjunta dos mapas de leitura do clorofilômetro e de produtividade do milho permite identificar áreas com menor capacidade de suprimento de nitrogênio pelo solo, e subsidia a delimitação de zonas para o manejo diferenciado do nitrogênio.

\section{Agradecimentos}

À Coordenação de Aperfeiçoamento de Pessoal de Nível Superior e ao Conselho Nacional de Desenvolvimento Científico e Tecnológico, pela concessão da bolsa; aos proprietários da Fazenda Alto Alegre e seus funcionários, pelo suporte prestado na condução do trabalho.

\section{Referências}

ALVES, V.M.C.; VASCONCELLOS, C.A.; FREIRE, F.M.; PITTA, G.V.E.; FRANÇA, G.E.; RODRIGUES FILHO, A.; ARAÚJO, J.M.; VIEIRA, J.R.; LOUREIRO, J.E. Milho. In: RIBEIRO, A.C.; GUIMARÃES, P.T.G.; ALVAREZ VENEGAS, V.H. (Ed.). Recomendações para o uso de corretivos e fertilizantes em Minas Gerais: 5a aproximação. Viçosa: Comissão de Fertilidade do Solo do Estado de Minas Gerais, 1999. p.314-316.

AMADO, T.J.C.; MIELNICZUK, J.; AITA, C. Recomendação de adubação nitrogenada para o milho no RS e SC adaptada ao uso de culturas de cobertura do solo sob sistema plantio direto. Revista Brasileira de Ciência do Solo, v.26, p.241-248, 2002.

AMARAL FILHO, J.P.R. do; FORNASIERI FILHO, D.; FARINELLI, R.; BARBOSA, J.C. Espaçamento, densidade populacional e adubação nitrogenada na cultura do milho. Revista Brasileira de Ciência do Solo, v.29, p.467-473, 2005.

ARGENTA, G.; SILVA, P.R.F. da; BORTOLINI, C.G.; FORSTHOFER, E.L.; STRIEDER, M.L. Relação da leitura do clorofilômetro com os teores de clorofila extraível e de nitrogênio na folha de milho. Revista Brasileira de Fisiologia Vegetal, v.13, p.158-167, 2001.

ARGENTA, G.; SILVA, P.R.F. da; MIELNICZUK, J.; BORTOLINI, C.G. Parâmetros de planta como indicadores do nível de nitrogênio na cultura do milho. Pesquisa Agropecuária Brasileira, v.37, p.519-527, 2002.

ARGENTA, G.; SILVA, P.R.F. da; SANGOI, L. Leaf relative chlorophyll content as an indicator parameter to predict nitrogen fertilization in maize. Ciência Rural, v.34, p.1379-1387, 2004.

BELOW, F.E. Fisiologia, nutrição e adubação nitrogenada do milho. Piracicaba: Potafos, 2002. p.7-12. (Informações Agronômicas, 99).

CAMBARDELLA, C.A.; MOORMAN, T.B.; NOVAK, J.M.; PARKIN, T.B.; KARLEN, D.L.; TURCO, R.F.; KONOPKA, A.E. Field-scale variability of soil properties in central Iowa soils. Soil Science Society of America Journal, v.58, p.1501-1511, 1994.

CANTARELLA, H.; DUARTE, A.P. Manejo da fertilidade do solo para a cultura do milho. In: GALVÃO, J.C.C.; MIRANDA, G.V. (Ed.). Tecnologias de produção do milho. Viçosa: UFV, 2004. p.139-182.

CARVALHO, J.R.P. de; SILVEIRA, P.M. da; VIEIRA, S.R. Geoestatística na determinação da variabilidade espacial de características químicas do solo sob diferentes preparos. Pesquisa Agropecuária Brasileira, v.37, p.1151-1159, 2002.

FANCELLI, A.L. Nutrição e adubação do milho. Piracicaba: USP, 2000. 43p.

FERGUSON, R.B.; HERGERT, G.W.; SCHEPERS, J.S.; GOTWAY, C.A.; CAHOON, J.E.; PETERSON, T.A. Site-specific nitrogen management of irrigated maize: yield and soil residual nitrate effects. Soil Science Society of America Journal, v.66, p.544-553, 2002.

FERNANDES, L.A.; VASCONCELLOS, C.A.; FURTINI NETO, A.E.; ROSCOE, R.; GUEDES, G.A. de A. Preparo do solo e adubação nitrogenada na produção de grãos e matéria seca e acúmulo de nutrientes pelo milho. Pesquisa Agropecuária Brasileira, v.34, p.1691-1698, 1999.

FERREIRA, D.F. SISVAR software: versão 4.6. Lavras: Ufla, 2003. Software.

KITCHEN, N.R.; HUGHES, D.F.; SUDDUTH, K.A.; BIRRELL, S.J. Comparison of variable rate to single rate nitrogen fertilizer application: corn production and residual soil $\mathrm{NO}_{3}-\mathrm{N}$. INTERNATIONAL CONFERENCE ON SITE-SPECIFIC MANAGEMENT FOR AGRICULTURAL SYSTEMS, 2., 1995, Madison. Proceeding. Madison: ASA, 1995. p.427-439.

MACHADO, P.L.O. de A.; BERNARDI, A.C. de C.; SILVA, C.A. (Ed.). Agricultura de precisão para o manejo da fertilidade do solo em sistema plantio direto. Rio de Janeiro: Embrapa Solos, 2004. 209p.

MAHMOUDJAFARI, M.; KLUITENBERG, G.J.; HAVLIN, J.L.; SCHWAB, A.P. Spatial variability of nitrogen mineralization at the field scale. Soil Science Society of America Journal, v.61, p.1214-1221, 1997.

MALAVOLTA, E.; VITTI, G.C.; OLIVEIRA, S.A. de. Avaliação do estado nutricional das plantas: princípios e aplicações. 2.ed. Piracicaba: Potafos, 1997. 319p. 
MENEGATTI, L.A.A.; MOLIN, J.P. Remoção de erros em mapas de produtividade via filtragem de dados brutos. Revista Brasileira de Engenharia Agrícola e Ambiental, v.8, p.126-134, 2004.

MINASNY, B.; MCBRATNEY, A.B.; WHELAN, B.M. Vesper version 1.6. Sydney: The University of Sydney: Australian Centre for Precision Agriculture, 2002. Disponível em: <http://www.usyd. edu.au/su/agric/acp >. Acesso em: 20 ago. 2006.

OLIVEIRA, S.A. de. Análise foliar. In: SOUSA, D.M.G. de; LOBATO, E. (Ed.). Cerrado: correção do solo e adubação. 2.ed. Planaltina: Embrapa Cerrados, 2004. p.245-256.

POVH, F.P.; MOLIN, J.P.; GIMENEZ, L.M.; PAULETTI, V.; MOLIN, R.; SALVI, J.V. Comportamento do NDVI obtido por sensor ótico ativo em cereais. Pesquisa Agropecuária Brasileira, V.43, p.1075-1083, 2008.

RAIJ, B. van; CANTARELLA, H.; QUAGGIO, J.A.; FURLANI, A.M.C. (Ed.). Recomendações de adubação e calagem para o estado de São Paulo. 2.ed. Campinas: IAC, 1996. 285p. (Boletim Técnico, 100).

RAMBO, L.; SILVA, P.R.F.; BAYER, C.; ARGENTA, G.; STRIEDER, M.L.; SILVA, A.A. da. Teor de nitrato como indicador complementar da disponibilidade de nitrogênio no solo para o milho. Revista Brasileira de Ciência do Solo, v.31, p.731-738, 2007.

RITCHIE, S.W.; HANWAY, J.J.; BENSON, G.O. Como a planta de milho se desenvolve. Piracicaba: Potafos, 2003. 20p. (Informações Agronômicas, 103).

SAS INSTITUTE. SAS: user's guide statistical: version 8.0. Cary: SAS Institute, 2000.

SILVA, E.C. da; BUZETTI, S.; GUIMARÃES, G.L.; LAZARINI, E.; SÁ, M.E. de. Doses e épocas de aplicação de nitrogênio na cultura do milho em plantio direto sobre Latossolo Vermelho. Revista Brasileira de Ciência do Solo, v.29, p.353-362, 2005.

SOUSA, D.M.G. de; LOBATO, E. Calagem e adubação para culturas anuais e semiperenes. In: SOUSA, D.M.G. de; LOBATO,
E. (Ed.). Cerrado: correção do solo e adubação. Planaltina: Embrapa Cerrados, 2004. p.283-315.

SOUZA, L.C.F.; GONÇALVES, M.C.; ALVES SOBRINHO, T.; FEDATTO, E.; ZANON, G.D.; HASEGAWA, E.K.B. Culturas antecessoras e adubação nitrogenada na produtividade do milho em plantio direto irrigado. Revista Brasileira de Milho e Sorgo, v.2, p.55-62, 2003.

SOUZA, L.V. de; MIRANDA, G.V.; GALVÃO, J.C.C.; ECKERT, F.R.; MANTOVANI, É.E.; LIMA, R.O.; GUIMARÃES, L.J.M. Genetic control of grain yield and nitrogen use efficiency in tropical maize. Pesquisa Agropecuária Brasileira, v.43, p.1517-1523, 2008.

TEDESCO, M.J.; GIANELLO, C.; BISSANI, C.A.; BOHNEN, H.; VOLKWEISS, S.J. Análises de solo, plantas e outros materiais. 2.ed. Porto Alegre: UFRGS, 1995. 175p.

VARVEL, G.E.; SCHEPERS, J.S.; FRANCIS, D.D. Ability for in-season correction of nitrogen deficiency in corn using chlorophyll meters. Soil Science Society of American Journal, v.61, p.1233-1239, 1997.

VIEIRA, S.R. Geoestatística em estudo de variabilidade especial do solo: In: NOVAIS, R.F. de; ALVAREZ VENEGAS, V.H.; SCHAEFER, C.E.G.R. (Ed.). Tópicos em ciência do solo. Viçosa: SBCS, 2000. p.1-55.

VITTI, G.C.; TEIXEIRA, L.H.B.; BARROS JÚNIOR, M.C. Diagnóstico da fertilidade do solo e adubação para alta produtividade de milho. In: FANCELLI, A.L.; DOURADO-NETO, D. (Ed.). Milho: estratégias de manejo para alta produtividade. Piracicaba: Esalq, 2003. p.174-197.

WEBSTER, R.; MCBRATNEY, A.B. On the akaike information criterion for choosing models for variograms of soil properties. Journal of Soil Science, v.40, p.493-496, 1989.

WELSH, J.P.; WOOD, G.A.; GODWIN, R.J.; TAYLOR, J.C.; EARL, R.; BLACKMORE, S.; KNIGHT, S.M. Developing strategies for spatially variable nitrogen application in cereals. Part I: Winter barley. Biosystems Engineering, v.84, p.481-494, 2003.

Recebido em 18 de outubro de 2008 e aprovado em 27 de fevereiro de 2009 